\title{
Transport Coefficients across Charged Mosaic Membrane
}

\author{
Wongkang Yang \\ Deparment of Chemistr, College of Natural Science, Dongguk Lniversitw, Gyeongin 780-714, Korea \\ Received November 17,2003
}

\begin{abstract}
In previous studies, charged mosaic membranes having two different fixed charges in the membrane matrix indicated unique transport behavior such as preferential material transport. In this study, the conposite charged mosaic membrane endurable to mechanical pressure in practical application was investigated from the same aspect of solute and solvent transport as before. $L p$ and $\omega$ estimated by taking account of active layer thickness were satisfactorily consistent with those in mosaic membrane without reinforcement. On the other hand. the reflection coefficient $s$ indicated the negative value that suggests preferential material transport.
\end{abstract}

Key Words : Charged mosaic membrane. Transport coefficient. Reflection coefficient

\section{Introduction}

Generally an ion exchange membrane can not permeate mostly cation or anion without applied current, while socalled charged mosaic membrane having two different fixed charges in membrane matrix can permeate both cation and anion in concentration gradient. In previous studies, ${ }^{1}$ we reported the transport behavior of solvent and solute across the charged mosaic membrane and indicated the unique characteristics in the charged mosaic membrane, such as the preferential solute transport. ${ }^{2}$ This means that one of the membrane parameters, $\sigma$ estimated from flux measurements indicates the negative value and the separation between solute and solvent or between electroly te and non-electroly te will be realized. ${ }^{3}$ In this study, the composite charged mosaic membrane with reinforcement as support film was investigated with respect to volume flux and solute flux. The results were compared to previous results of the charged mosaic membrane without reinforcement and discussed. ${ }^{2}$

\section{Experimental Section}

Materials. All the mosaic membranes and composite mosaic membranes in this studies were kindly supplied by Dainichi Seika Industry Co. Ltd. Japan. The detailed procedure to make the membrane preparation were described in literatures. ${ }^{5}$ Other reagents were used without further purification. Doubly distilled water was used to prepare an aqueous solution.

Transport studies. The flux measurements were carried out by using two glass cells. The membranes were tightly inserted between two cells by using silicon rubbers in order to axoid leak of solution from the contact surfaces. Each cell volume is $25 \mathrm{~mL}$ and the membrane area is $3.14 \mathrm{~cm}^{2}$. Temperature of glass cells were kept at $25^{\circ} \mathrm{C}$ by circulating constant water surround the cells during experiment. Time changes of volume or concentration in cell 1 or cell 2 were measured using graduated glass capillary or pencil-type

e-mail: yangwk àdongguk ac.ks conductance cell. Volume flux and solute flux were evaluated from volume change vs. time or concentration change versus time by taking account membrane area, $3.14 \mathrm{~cm}^{2}$. The volume flux and solute flux were examined in different two apparatus. system I and system II, separately.

Apparatus. System I: The sucrose solution $\left(0.5 \mathrm{moldm}^{-3}\right)$ and distilled water were placed in celll and cell2, respectively. Furthermore. in order to examine the added salt effects. equal amounts of $\mathrm{KCl}$ were inserted into both cells and the $\mathrm{KCl}$ concentrations were changed from 0 to 0.5 moldm ${ }^{-3}$.

Sustem $I$ : The $\mathrm{KCl}$ solution and distilled water were inserted into cell $\mathrm{l}$ and cell 2 . respectively, and $\mathrm{KCl}$ concentrations were changed from 0.01 to 1 moldm ${ }^{-3}$.

\section{Results and Discussion}

The volume change versus time in system I using sucrose as impermeable solute indicated linearity. ${ }^{6}$ All the other relations with various added $\mathrm{KCl}$ concentrations in system I also indicated linearity. This ${ }^{6}$ means the systems are in steady state within examined time. ${ }^{7}$ Similarly. the volume change and $\mathrm{KCl}$ concentration change versus time in system II were obtained as linear relations being steady state. However. the direction of volume change in system II was opposite to that in șystem I.

Filtration coefficient, $L p$. In experimental section. taking account of membrane area for the slopes of straight lines. one can obtain volume flux and solute flux. In addition to that according to equations in previous studies, three important membrane parameters. $L p, \sigma$ and $\omega$ can be estimated ${ }^{8}$. $L p$ is filtration coefficient that means water permeability or water affinity with membrane and is given in Figure 1 along with those about the charged mosaic membrane without reinforcement. In Figure 1 the obtained $L p$ 's values were given as added $\mathrm{KCl}$ concentrations and the result indicated the water affinity in membrane was not affected by existence of electrolytes in outer solutions. On the other hand. in comparison with charged mosaic membrane without reinforcement. $L p^{\prime}$ s values in composite membrane became 


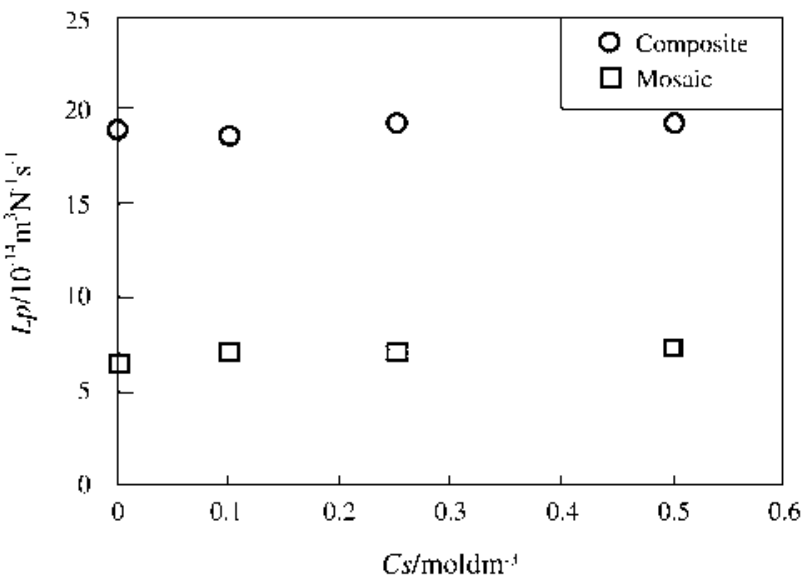

Figure 1. Dependence of water permeabilities $L_{p}$, on salt concentralion in system I.

larger than that in the mosaic membrane without reinforcement. This seems to be inconsistency because material transports depend on membrane thickness and the values are almost the same in both membranes. The result will be discussed later along with that in solute permeability."

Reflection coefficient, $\sigma$ and salt permeability coefficient, $\boldsymbol{\omega}$. In Figures $2 \mathrm{a}$ and $2 \mathrm{~b}$, the reflection coefficients and salt permeability coefficients estimated from the results in system II were given along with the previous results.

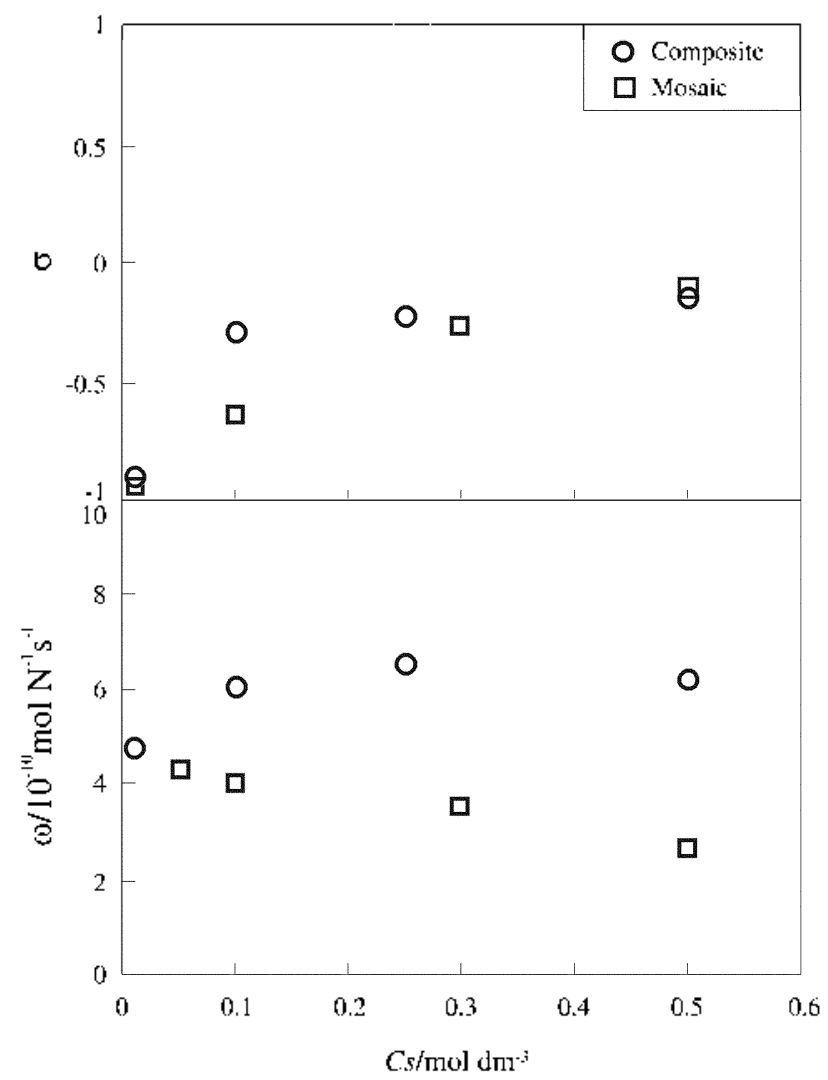

Figure 2. Reflection cocfficients. $\sigma$ (above) and salt permeabilities. $\omega$ (below). through charged mosaic membrane on nonequilitrium thermody namics.
Regarding $\sigma$, the values were almost the same as those in previous studies and indicated the negative values in the examined concentration ranges as seen in Figure $2 \mathrm{a}$. Negative $\sigma$ implies the preferential salt transport over solvent transport and is very important for practical pressure dialysis. ${ }^{10}$ On the other hand, $\omega$ 's values indicated the different values between two charged mosaic membranes and present membranes were more permeable than previous membrane for water transport as seen in Figure $2 b$. Those two values, $L p$ in Figure 1 and $\omega$ in Figure $2 \mathrm{~b}$ suggest the physical quantities are closely related to active layer in total membrane thickness. From the fact mentioned above, the equations used for evaluation of $L p, \sigma$ and $\omega$ do not contain the information about active layer thickness.

The phenomenological equation. According to Kedem and Katchalsky," membrane parameters, $L p, \sigma$ and $\omega$ in appropriate experimental conditions were given as follows, eqs. (1), (2) and (3).

$$
\begin{aligned}
& L_{p}=-\frac{J v_{\Delta P} 0}{\Delta \Pi}, \\
& \sigma--\frac{J v_{\Delta /}^{\prime}=0}{L p \Delta \Pi}, \\
& \omega=\frac{\Delta \Pi}{J_{S / S-0, N^{\prime}-0}} .
\end{aligned}
$$

The membrane parameters in Figure 1 and Figure 2 were estimated using Eqs. (1)-(3). As described above, the improved equation containing information about active layer thickness would be convenient to explain the discrepancy between present and previous results. The equations allowing for the membrane thickness will be given as follows,

$$
\begin{aligned}
& J_{v}-L p^{\prime}\left(\frac{d P}{d x}-\sigma \frac{d \Pi}{d x}\right), \\
& J_{s}=C^{\prime \prime}(1-\sigma) J_{v}-\omega^{\prime} \frac{d \Pi}{d x} .
\end{aligned}
$$

Integrating over membrane thickness, one can obtain the following equations,

$$
\begin{aligned}
& J v=\frac{L p^{\prime}}{\delta}(\Delta P-\sigma \Delta \Pi), \\
& J_{S}-C_{s}^{\prime \prime}\left(\begin{array}{ll}
1 & \sigma
\end{array}\right) J_{v} \cdot \frac{\omega^{\prime}}{\delta} \Delta \Pi
\end{aligned}
$$

where $L p^{\prime}$ and $\omega^{\prime}$ mean coeflicients including membrane information and $\delta$ is membrane thickness. Compared Eqs. (1)-(3) with Eqs. (6)-(7), one can oblain the relation between present and previous $L_{p}$ or $\omega$ as follows,

$$
\begin{aligned}
& L p^{\prime}=L p \cdot \delta, \\
& \omega^{\prime}-\omega \cdot \delta,
\end{aligned}
$$

where $L p^{\prime}$ or $\omega^{\prime}$ are parameters taking account of membrane thickness. If active layer consist of same materials, the values should be same irrespective of membrane with or 
Table 1. Lp and Active Layer Thickness or membranc thickness

\begin{tabular}{ccc}
\hline & $L p / 10^{1+} \mathrm{m}^{3} \mathrm{~N}^{\mathrm{s}} \mathrm{s}^{3}$ & Thickness $\delta / \mu \mathrm{m}$ \\
\hline Raw & 7.07 & 50 \\
Composite & 19.1 & 18.5 \\
\hline
\end{tabular}

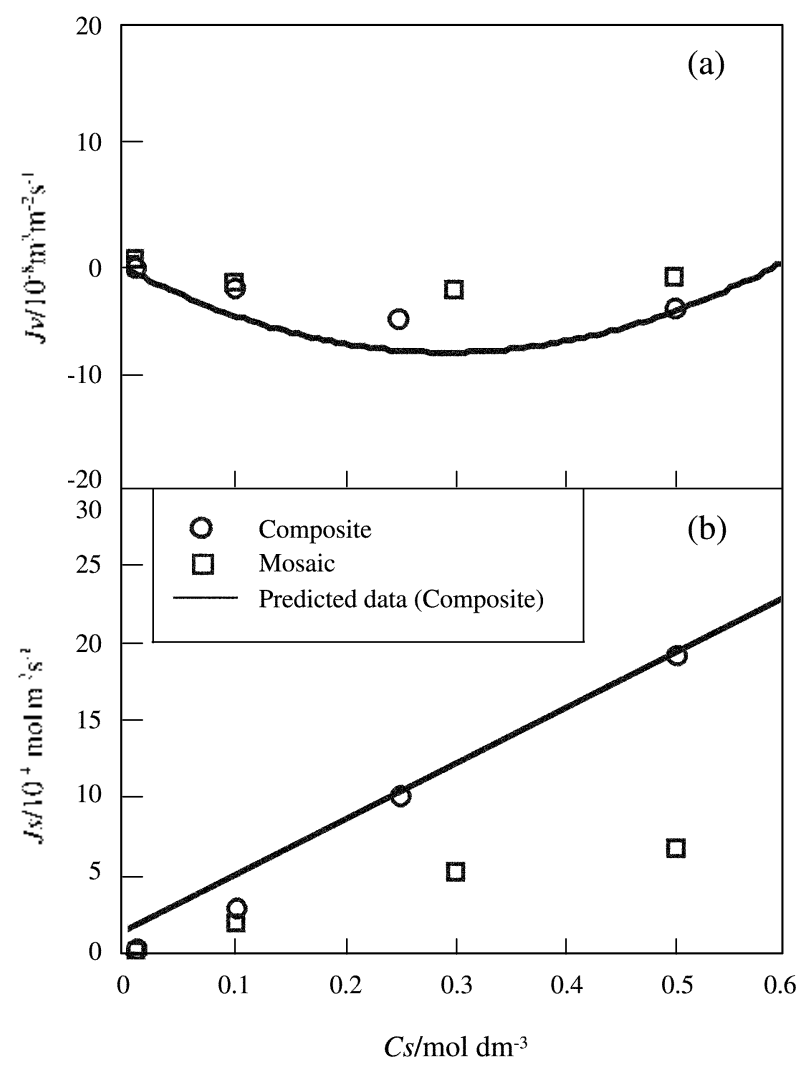

Figure 3, (a) and (b) Comparison of experimental data with predicted data of composite membrane in volume and salt fluxes.

without reinforcement. 'Therefore, for reference to $L p^{\prime}$ ' value in $50 \mu \mathrm{m}$ thickness of active layer, effective thickness of active layer in composite membrane was determined as 18.5 $\mu \mathrm{m}$ and given in Table 1 . When one allow for the thickness of active layer as $18.5 \mu \mathrm{m}, L p$ or $\omega$ indicating large values would be reasonable, that is, the thinner the membrane thickness, the larger the volume or salt flux. ${ }^{11}$

Reproduction of $J v$ and $J \boldsymbol{s}$. In order to verify the active layer thickness of composite membrane, $J v$ and.$J$ about the composite membrane was reproduced from the previous result using $18.5 \mu \mathrm{m}$ thickness and given in Figures $3 \mathrm{a}$ and 3b. As seen in Figure 3, the predicted solid lines were satisfactorily accord to the experimental results in composite charged mosaic membrane. In conclusion, the composite charged mosaic membrane was proved to exhibit the excellent transport performance. ${ }^{12}$ The potential application to pressure dialysis was expected.

\section{Conclusions}

Salt and water transport under the mixed $\mathrm{KCl}$ and sucrose solution system were investigated. Two kind of measurements, volume flux and solute flux, were performed. Volume fluxes across charged mosaic membrane could be classified into osmotic flow of water due to $\mathrm{KCl}$ concentration difference, water flow dragged by $\mathrm{KCl}$ diffusion, and osmotic flow water due to sucrose and they were found to be additive. In addition, it was elucidated that the presence of non-electrolyte solution can easily change the direction and the magnitude of volume fluxes. By the results, it was suggested that efficient salt enrichment can be performed by controlling the direction of the solvent osmosis and one can expect negative salt rejection same as reverse osmosis.

Acknowledgement. This work was supported by a program of Dongguk University Research Fund and a grant No. JR 020 from Korea-Japan Basic Scientific Program of KOSEF program and author would like to appreciate Dr. A. Yamauchi at Kyushu University (Japan) for the helpful conductivity measurement.

\section{References}

1. Yamauchi. A.: Tateyama. I.: Etoh. B.: Takizawa. M.: Sugito. Y.: Doi, S. J. Memb. Sci. 2000. /73. 275.

2. Iiukuda T:: Yamaluchi. A. Bufl. Chem. Soc. Jpm. 2000.73. 2729.

3. Iukuda T.: Yamauchi. A. Btfl. Soc. Sea llater Sci. Jph. 2002, 56. 33.

4. Fukuda. T.: Yang. W.: Yamauchi. A. f. Memh. Sci. 2003. 212. 255 .

5. Nakamura M.: Fukutomi, T.: Takizawa M.: Sigito. Y.: Doi, S. IISP-5543045 \& Japan Kokai. 10-087855.

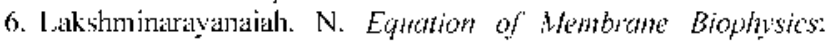
Academic press: Inc.. 1984.

7. Weinstein. I. N.: Caplan. S. R. Science 1968. /6/. 70.

8. Katchalsky. A.: Curran. P. F. Aon-tanilibrium Thermodymamics in Biophnsic:s: l larvard University Press: 1965.

9. Kedem. O.: Kattelalsky A. Trans. Faraden Soc. 1962.59. 1931.

10. Sollner. K. Biochem. Z. 1932. 244. 370.

11. Yang. W.: Yamauchi. A.: Kimizuka. H. J. Membrane So. 1992. 70. 277

12. Jcong. S.: Lec. W.: Yang. W. Butl. Koretn (hem. Soc. 2003. 212. 937. 